\title{
Solitary extrapulmonary lymphangioleiomyomatosis of the liver: A case report and literature review
}

\author{
WEIWEI FU ${ }^{1}$, YUJUN LI ${ }^{1}$, HONG LI $^{1}$, PING YANG $^{2}$ and XIAOMING XING ${ }^{1}$ \\ ${ }^{1}$ Department of Pathology, The Affiliated Hospital of Qingdao Medical College, \\ Qingdao University, Qingdao, Shandong 266003; ${ }^{2}$ Department of Pathology, \\ The Affiliated Yantai Yuhuangding Hospital of Qingdao University, \\ Yantai, Shandong 264000, P.R. China
}

Received March 17, 2015; Accepted April 11, 2016

DOI: $10.3892 /$ etm.2016.3502

\begin{abstract}
Lymphangioleiomyomatosis (LAM) is a progressive disorder of unknown etiology that predominantly affects the lungs, and the resulting respiratory failure can be fatal. Extrapulmonary LAM is a rare disease that often occurs concurrently with pulmonary LAM. Usually, extrapulmonary LAM presents as a localized, well-circumscribed mass. Due to the rare occurrence of extrapulmonary LAM and its atypical location, extrapulmonary LAM is often difficult to diagnose prior to surgery. The present study describes the clinicopathological features of primary liver LAM in a 26-year-old woman. To the best of our knowledge, the present study presents the first case of a primary solitary extrapulmonary form of LAM in the liver.
\end{abstract}

\section{Introduction}

Lymphangioleiomyomatosis (LAM) is a progressive disorder that predominantly affects the lungs, and the resulting respiratory failure can be fatal (1). The typical histology of LAM shows a proliferation of smooth muscle arranged in fascicular, trabecular, and papillary patterns associated with slit-like vascular channels (2). Pulmonary LAM can occur sporadically or in association with the inherited hamartoma syndrome tuberous sclerosis (TSC) $(3,4)$.

Extrapulmonary LAM is a rare disease that is usually presented as a localized, well-circumscribed mass (5). The primary sites for LAM include the pancreas, retroperitoneum, pelvis, mediastinum, kidney hilus, uterus and mesentery (6). The clinical features of extrapulmonary lymphangioleiomyomatosis are a palpable abdominal mass, abdominal pain, and

Correspondence to: Dr Xiaoming Xing, Department of Pathology, The Affiliated Hospital of Qingdao Medical College, Qingdao University, 16 Jiangsu Road, Qingdao, Shandong 266003, P.R. China E-mail: edithxing@hotmail.com

Key words: lymphangioleiomyomatosis, extrapulmonary, solitary, liver chylous ascites (7). Differential diagnoses should include lymphoma, schwannoma, paraganglioma at the para-aortic area, and metastatic tumors in the lymph nodes (2). Due to the rare occurrence of extrapulmonary LAM and its atypical location, extrapulmonary LAM is often difficult to diagnose, especially prior to surgery. To the best of our knowledge, the present study reports the first case of primary LAM presenting as a liver mass.

\section{Case report}

Case summary. The present study was approved by the Ethics Committee of the Qingdao University Medical College (Qingdao, China). Written informed consent was obtained from the patient's family. In 2009, a 26-year-old woman presented a liver lesion of $\sim 6 \mathrm{~cm}$ diameter during a routine examination at Jiaozhou People's Hospital of Qingdao (Qingdao, China). The liver lesion was diagnosed as a liver cyst following a computed tomography (CT) scan of the abdomen. The patient exhibited no clinical symptoms until April 2012. Enhanced abdominal CT scans showed that the mass was located in the left lateral hepatic lobe with $5.7 \times 7 \mathrm{~cm}$ enhancement and uneven density (Fig. 1). The radiological differential diagnosis included left lateral hepatic adenoma or focal nodular hyperplasia. Subsequently, a hepatic lobectomy was performed in May, and the patient was discharged after one week.

Pathologic findings. Gross examination demonstrated that the liver resection specimen measured $15 \times 10 \times 4 \mathrm{~cm}$. After the specimen was cut longitudinally into 1-cm lobe sections, the liver lobe section exhibited a cystic solid gray-yellow mass measuring $6 \times 5 \mathrm{~cm}$. The size of the cysts were $0.5-2.5 \mathrm{~cm}$.

Histological examination was subsequently performed. Following fixation in $10 \%$ neutral-buffered formalin, paraffin embedding, sectioning and staining with hematoxylin and eosin, examination under an Olympus BX51 microscope (Olympus Corporation, Tokyo, Japan) demonstrated that the tumor exhibited the proliferation of spindle cells arranged along slit-like channels lined with flattened endothelium (Fig. 2A). These spindle cells were uniform with abundant granular eosinophilic cytoplasm and lightly stained nuclei with a smooth nuclear membrane and inconspicuous nucleoli, which were organized 


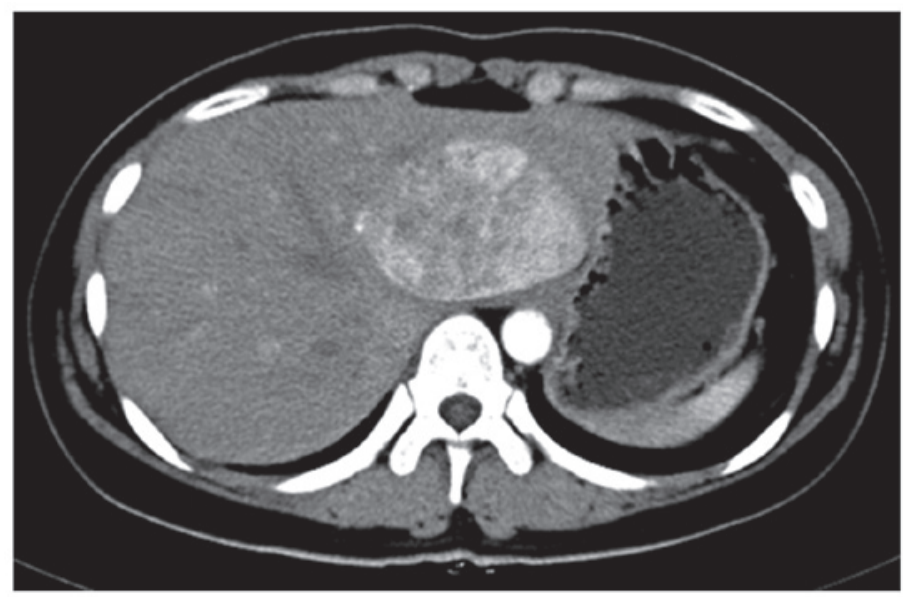

Figure 1. Enhanced computed tomography scan showing a $5.7 \times 7 \mathrm{~cm}$ enhancement and uneven density involving the left lateral hepatic.
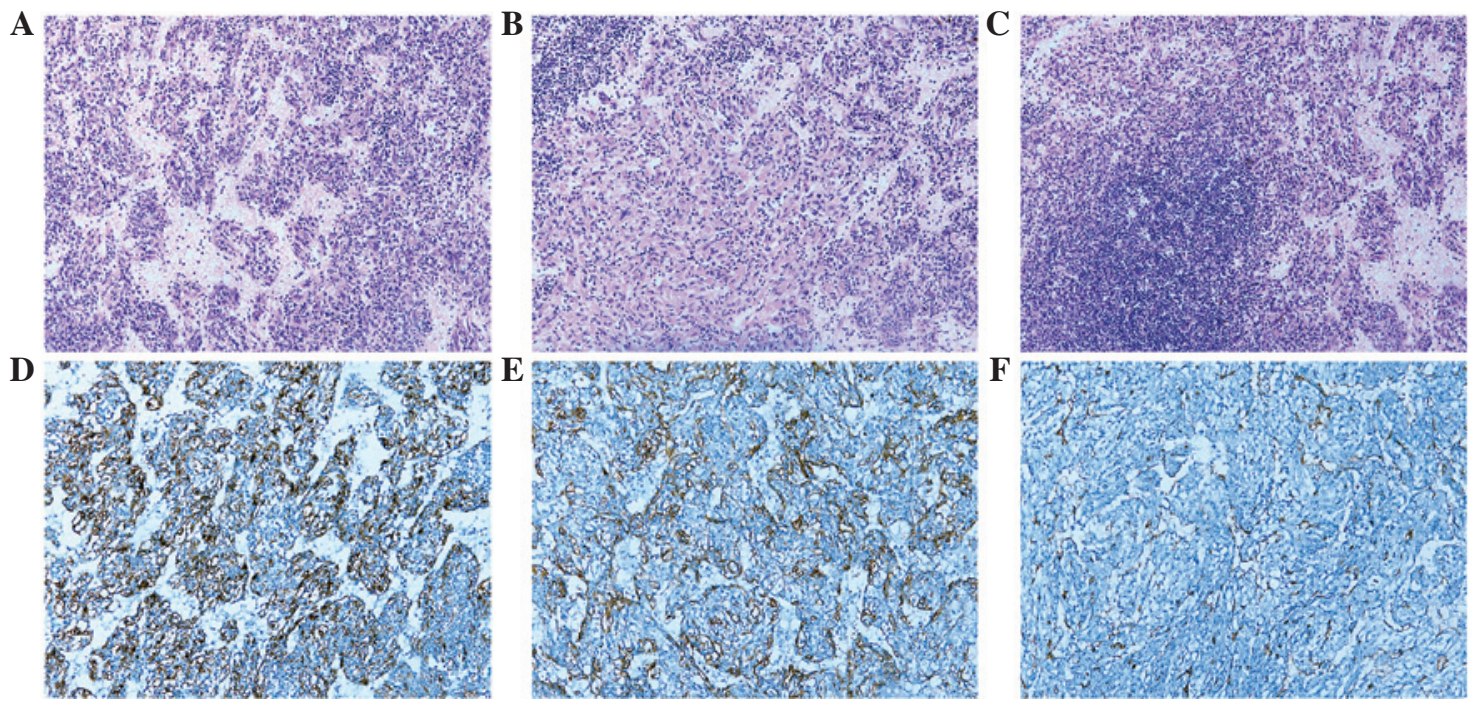

Figure 2. Immunohistochemical staining of the liver tumor. (A) The tumor is composed of spindle cells arranged along slit-like channels lined with flattened endothelium (H\&E staining; magnification, x200). (B) The tumor cells are spindles with abundant eosinophilic cytoplasm and lightly stained nuclei with a smooth nuclear membrane and inconspicuous nucleoli (H\&E staining; magnification, x200). (C) In certain areas, the aggregation of lymphoid cells can be observed (H\&E staining; magnification, x200). (D and E) The tumor cells are positive for human melanoma black-45, MelanA and smooth muscle actin (magnification, x100); (F) The endothelia are positive for CD31 (Envision x100). H\&E, hematoxylin and eosin.

in fascicular, trabecular and anastomosing cord-like growth pattern (Fig. 2B). In some areas, the aggregation of lymphoid cells was observed (Fig. 2C). Images were captured using an XV Image Processing System (Olympus Corp.).

Immunohistochemistry. Immunohistochemical staining was performed on paraffin-embedded tissue sections ( $5 \mu \mathrm{m})$ using the EnVision method (8). In brief, sections were deparaffinized and pretreated in $10 \mathrm{mM}$ citrate buffer solution $(\mathrm{pH} 6.0)$. Sections were treated with $0.3 \%$ hydrogen peroxide in methanol for $30 \mathrm{~min}$ to block the endogenous peroxidase, and were subsequently washed in phosphate-buffered saline. Sections were incubated overnight at $4^{\circ} \mathrm{C}$ with: Human melanoma black-45 [working solution (WS); HMB-45],Ki67 (1:200; K-2), Desmin (WS; D33) and smooth muscle actin (SMA; 1:300; 1A4) mouse monoclonal antibodies; MelanA (WS; A103) and CD31 (WS; EP78) rabbit monoclonal antibodies; and S-100 rabbit polyclonal antibody (WS; ZA0225; all purchased from
ZSGB-BIO, Beijing, China). Following washing, section were incubated with the secondary antibody (WS; PV6000; ZSGB-BIO) for $20 \mathrm{~min}$ at $37^{\circ} \mathrm{C}$. A positive reaction was observed using diaminobenzidine, followed by counterstaining with hematoxylin. Immunohistochemistry demonstrated that the spindle cells were positive for HMB-45 (Fig. 2D), MelanA and SMA (Fig. 2E), but negative for Desmin and S-100. The endothelium was positive for CD31 (Fig. 2F). Proliferation index with Ki-67 showed $2 \%$ positivity in the tumor cells. The patient did not receive any further treatment. No other lesions were detected at biannual pulmonary and abdominal CT scans, and the patient is well 30 months post-surgery.

\section{Discussion}

LAM is a rare neoplastic disease which predominantly affects women with a mean age of onset of 37 years. The majority of LAM cases occur in the lung and exhibit multiple cysts (9). 
Patients with pulmonary LAM present progressive dyspnea, and pneumothorax and chylous pleural effusion. In contrast to pulmonary LAM, extrapulmonary LAM lesions are rare and manifest as localized lesions (10). According to previous reports, the primary sites of extrapulmonary LAM include the posterior mediastinum, the upper retroperitoneal areas near the abdominal aorta and the pelvic cavity (6). However, to the best of our knowledge, the occurrence of LAM in the liver has yet to be reported. Only one case of abdominal LAM that presented multiple cysts involving the liver was described by Possekel et al (11) in a 23-year-old patient. The present case is the first extrapulmonary LAM presenting as a liver mass without involving other organs.

LAM, together with angiomyolipoma, clear-cell (sugar) tumor of the lung and clear-cell myomelanocytic tumor of the falciform ligament/ligamentum teres belongs to the perivascular epitheloid cell tumor (PEComa) family (12). PEComas has been described as 'a mesenchymal tumour composed of histologically and immunohistochemically distinctive perivascular epithelioid cells' by the World Health organization (13).

Histologically, LAM is characterized by the proliferation of immature smooth muscle and perivascular epitheloid cells (LAM cells) (14). These cells are spindle-shaped or round with lightly stained nuclei with a smooth nuclear membrane and inconspicuous nucleoli and are arranged in a haphazard manner or in fascicular, trabecular, and papillary patterns associated with slit-like vascular channels (2). Immunohistochemically, the tumor cells exhibited reactivity against SMA and melanocytic (HMB-45 and MelanA) markers (15). The present case exhibited the distinct morphology of classical LAM which showed the proliferation of smooth muscle spreading along the lymphatic channels. Immunostaining revealed the tumor cells were positive for HMB-45, MelanA and SMA. Although with distinct morphological and immunophenotypic features extrapulmonary LAM can be easily diagnosed, it is difficult to diagnose by radiology, especially in the absence of accompanying pulmonary LAM. Radiologically, the present case was initially diagnosed as hepatic adenoma and focal nodular hyperplasia.

LAM can be divided into a sporadic form and a tuberous sclerosis-associated form (4). Both forms can cause pulmonary symptoms. Extrapulmonary LAM has the same morphological features as pulmonary LAM and may occur concurrently with it or precede it (12). Matsui et al (6) described 22 cases of extrapulmonary LAM, 13 of which exhibited pulmonary LAM within 2 years. Furthermore, 3/6 cases described by Song et al (5) also exhibited concurrent pulmonary LAM and extrapulmonary LAM. However, the present case showed a localized liver mass without a pulmonary manifestation prior to or following the surgical procedure, which is similar to the cases described by Han et al (7) and Singh et al (12).

To date, sufficient data has not been acquired regarding the prognosis of extrapulmonary LAM. According to the report of Matsui et al (6), only one patient succumbed to pulmonary LAM 7 years following the initial diagnosis, whereas the remaining 16 patients were alive at $1-13$ years. The patient in the present case report is alive without any progression 4 years following the initial surgical procedure. As extrapulmonary LAM tends to lead to pulmonary LAM or angiomyolipomas (6), a close follow-up is necessary.
Studies on the therapeutic strategies for extrapulmonary LAM have been limited. Current studies provide the foundation for novel therapeutic strategies for LAM. LAM occurs in $\sim 30 \%$ of woman with TSC (16-18), and has therefore been well-described as a complication of tuberous sclerosis associated with mutations in the tuberous sclerosis genes TSC1 and TSC2 (19). Specifically, TSC genes (TSC1 and TSC2) have an important role in the regulation of the cell cycle via the mammalian target of rapamycin (mTOR) signaling pathway (20). Sirolimus, also called rapamycin (an mTOR inhibitor) blocks the mTOR signaling pathway and restores homeostasis in the LAM cell (18). Possekel et al (11) described a 23-year-old woman with abdominal manifestations of LAM, who received sirolimus therapy and showed clinical benefit. On the other hand, anti-estrogenic therapy, such as oophorectomy and progesterone, has also been demonstrated to be an alternative way to treat LAM (21). Application of hormone therapy in extrapulmonary LAM had been reported, whereas the guidelines of the European Respiratory Society on the diagnosis and treatment of LAM (16) published in 2010, did not recommend the standard use of progesterone.

To conclude, the present study presents a case of localized liver LAM with fibrous capsule in a 26-year-old woman with no symptoms. Radiological examination revealed a potential hepatic adenoma or focal nodular hyperplasia. However, pathological examination revealed the liver lesion to be a histologically and immunohistochemically classic LAM. Therefore, for female patients in whom liver masses are observed, extrapulmonary LAM should be considered in the differential diagnosis.

\section{Acknowledgements}

The present study was supported by the Natural Science Foundation of China (grant no. 81201947).

\section{References}

1. Li C, Lee PS, Sun Y, Gu X, Zhang E, Guo Y, Wu CL, Auricchio N, Priolo C, Li J, et al: Estradiol and mTORC2 cooperate to enhance prostaglandin biosynthesis and tumorigenesis in TSC2-deficient LAM cells. J Exp Med 211: 15-28, 2014.

2. Jaiswal VR, Baird J, Fleming J, Miller DS, Sharma S and Molberg K: Localized retroperitoneal lymphangioleiomyomatosis mimicking malignancy. A case report and review of the literature. Arch Pathol Lab Med 127: 879-882, 2003.

3. Johnson SR: Lymphangioleiomyomatosis. Eur Respir J 27: 1056-1065, 2006.

4. Taveira-DaSilva AM, Pacheco-Rodriguez G and Moss J: The natural history of lymphangioleiomyomatosis: Markers of severity, rate of progression and prognosis. Lymphatic Res Biol 8: 9-19, 2010.

5. Song DH, Choi IH, Ha SY, Han KM, Lee JJ, Hong ME, Choi YL, Jang KT, Song SY, Yi CA and Han J: Extrapulmonary lymphangioleiomyoma: Clinicopathological analysis of 4 cases. Korean J Pathol 48: 188-192, 2014.

6. Matsui K, Tatsuguchi A, Valencia J, Yu Zx, Bechtle J, Beasley MB, Avila N, Travis WD, Moss J and Ferrans VJ: Extrapulmonary lymphangioleiomyomatosis (LAM): Clinicopathologic features in 22 cases. Hum Pathol 31: 1242-1248, 2000.

7. Han JM, Lee KH, Kim SJ, Rhim CC, Park YH, Kang JB and Jeon SY: A case of lymphangioleiomyomatosis originated in the pelvic cavity. J Gynecol Oncol 19: 195-198, 2008.

8. Wenjuan Y, Yujun L and Ceng Y: Association of single nucleotide polymorphisms of beta2-adrenergic receptor gene with clinicopathological features of pancreatic carcinoma. Acta Histochem 115: 198-203, 2013. 
9. Lu SH, Hou YY, Tan YS, Xu JF, Zeng HY, Sujie AK, Wang XD and Bai CX: Clinical and histopathological alterations of lymphangioleiomyomatosis in 14 Chinese patients. Chinese Med J 122: 1895-1900, 2009.

10. Kitaichi M, Nishimura K, Itoh $\mathrm{H}$ and Izumi T: Pulmonary lymphangioleiomyomatosis: A report of 46 patients including a clinicopathologic study of prognostic factors. Am J Resp Crit Care Med 151: 527-533, 1995.

11. Possekel AK, Katenkamp D, Brambs HJ and Pauls S: Lymphangioleiomyomatosis: Solitary abdominal manifestation (2009: 9b). Eur Radiol 19: 3015-3018, 2009.

12. Singh M, Saroha V, Wadhwa R, Khurana N and Kakkar AK: Solitary lymphangioleiomyoma of pancreas mimicking pancreatic pseudocyst-a case report. J Gastrointest Cancer 43 336-339, 2012.

13. Foipe AL: Neoplasms with perivascular epithelioid cell differentiation In: World Health organization classification of tumours: Pathology and genetics of tumours of soft tissue and bone. Fletcher CD, Unni KK, Mertens F, et al (eds): LARC Press, Lyon, pp221-222, 2002.

14. Grzegorek I, Drozdz K, Podhorska-Okolow M, Szuba A and Dziegiel P: LAM cells biology and lymphangioleiomyomatosis. Folia Histochem Cytobiol 51: 1-10, 2013.

15. Hornick JL and Fletcher CD: PEComa: What do we know so far? Histopathology 48: 75-82, 2006.
16. Johnson SR, Cordier JF, Lazor R, Cottin V, Costabel U, Harari S, Reynaud-Gaubert M, Boehler A, Brauner M, Popper H, et al: European Respiratory Society guidelines for the diagnosis and management of lymphangioleiomyomatosis. Eur Respir J 35: 14-26, 2010.

17. Casanova A and Ancochea J: Lymphangioleiomyomatosis: new therapeutic approaches. Arch Bronconeumol 47: 579-580, 2011 (In Spanish).

18. Derweduwen AM, Verbeken E, Stas M, Verschakelen J, Coolen J, Verleden G and Wuyts W: Extrapulmonary lymphangioleiomyomatosis: A wolf in sheep's clothing. Thorax 68: 111-113, 2013.

19. McCormack FX: Lymphangioleiomyomatosis: A clinical update. Chest 133: 507-516, 2008.

20. Goncharova EA, Goncharov DA, Eszterhas A, Hunter DS Glassberg MK, Yeung RS, Walker CL, Noonan D, Kwiatkowski DJ, Chou MM, et al: Tuberin regulates p70 S6 kinase activation and ribosomal protein S6 phosphorylation. A role for the TSC2 tumor suppressor gene in pulmonary lymphangioleiomyomatosis (LAM). J Biol Chem 277: 30958-30967, 2002.

21. Ohori NP, Yousem SA, Sonmez-Alpan E and Colby TV: Estrogen and progesterone receptors in lymphangioleiomyomatosis, epithelioid hemangioendothelioma and sclerosing hemangioma of the lung. Am J Clin Pathol 96: 529-535, 1991. 